\title{
The Effect of Creating a Moderate Stenosis on the Localization of Intimal Thickening in the Common Carotid Artery of the Rabbit Fed on a Cholesterol-Rich Diet*
}

\author{
Shigeo WADA**, Makoto KOUJIYA*** \\ and Takeshi KARINO**
}

\begin{abstract}
The effect of a locally disturbed flow on intimal thickening was studied by creating a moderate axisymmetric stenosis (mean diameter reduction: 26\%) in common carotid arteries of the rabbit and feeding a cholesterol-rich diet. In eight of nine specimens obtained from four rabbits, intimal thickening occurred at the distal side of the stenosis, mainly in a restricted region which extended from the apex of the stenosis to the distal end, the point of the maximum thickness locating approximately a half of the vessel diameter downstream of the apex of the stenosis. The result was compared with the distributions of wall shear stress and surface concentration of low-density lipoproteins (LDL) which were calculated using a theoretical model of blood flow and LDL transport through the stenosed artery. It was found that the site of intimal thickening well corresponded to the region where wall shear stress was relatively low and surface concentration of LDL was locally elevated. These results suggest that blood flow plays an important role in the localized pathogenesis and development of intimal thickening by locally augmenting concentration polarization of LDL at a blood/endothelium boundary.
\end{abstract}

Key Words: Biomechanics, Fluid Dynamics, Blood Flow, Mass Transport, Stenosis, Intimal Thickening, Lipoproteins, Concentration Polarization, Rabbit, Common Carotid Artery

\section{Introduction}

Atherosclerosis is a degenerative vascular disease that occurs in relatively large arteries by progressive thickening of the intima of the vessel wall and its eventual hardening. Accumulation of atherogenic lowdensity lipoprotein (LDL), which is a main carrier of cholesterol in blood, within the arterial intima is regarded as the first step leading to the initiation of atherosclerosis ${ }^{(1),(2)}$. It has been shown that atherosclerotic changes of an arterial wall develop preferen-

* Received 1st June, 2001

** Research Institute for Electronic Science, Hokkaido University, North 12, West 6, North District, Sapporo, Hokkaido 060-0812, Japan. E-mail : wada @bfd. es.hokudai.ac.jp

*** R \& D Center, Nikkiso Co., Ltd., 498-1 Shizutani, Haibara-cho, Haibara-gun, Shizuoka 421-0496, Japan tially at certain sites such as the inner wall of curved segments and the outer walls of bifurcations of large and medium-sized arteries in the human circulatory system $^{(3)-(5)}$ where flow is disturbed and endothelial cells are exposed to relatively low shear stresses ${ }^{(6)-(10)}$. Involvement of blood flow has also been found in the progression of intimal thickening at anastomotic junctions between the host artery and a synthetic or autologous vein $\mathrm{graft}^{(11)}$ and distal to a stenosis which was artificially created in an artery of an animal by winding a suture thread ${ }^{(12)}$. Therefore, it is suspected that fluid mechanical factors are involved in the pathogenesis and localization of these vascular diseases by affecting the accumulation of lipoproteins within the intima of arteries. However, the precise mechanism has not been elucidated yet.

To explain the localized genesis and development of these vascular diseases, we previously proposed flow-dependent concentration polarization of lipo- 
proteins at a blood/endothelium boundary which is a phenomenon we found through a series of theoretical and experimental studies on mass transport of lipoproteins in blood flowing through an artery to its vessel wall where a monolayer of endothelial cells acts as a barrier to macromolecules such as LDL to infiltrate into the subendothelial space $\mathrm{s}^{(13)-(15)}$. The results obtained so far for the cases of a straight portion and curved segment of an artery showed that due to a semipermeable nature of an arterial wall to plasma, accumulation of lipoproteins occurs at a blood/endothelium boundary, and the concentration of lipoproteins at the luminal surface of the vessel wall varies as a function of wall shear rate, leading to uneven distribution of LDL concentration on the vessel wall.

The present study was designed to investigate the effect of locally disturbed flows on the localization of intimal thickening by carrying out animal experimentations. To do so, we created a moderate stenosis in the common carotid artery of the rabbit, kept the animal for several weeks by feeding a cholesterol-rich diet, and measured the precise location and the extent of intimal thickening formed in the artery after harvesting it. Furthermore, theoretical calculations of blood flow and the concentration of LDL at the luminal surface of the vessel were also carried out using an axisymmetric model of stenosed arteries. Based on the results of these experimental and computational studies, we examined the relationship among the wall shear stress, the surface concentration of LDL, and the thickness of the intima at sites where intimal thickening occurred in the artery to elucidate the role of blood flow in the localization of intimal thickening in the arterial system.

\section{Experimental Study}

\section{1 Materials}

Common carotid arteries of male Japanese white rabbits weighing from 3.1 to $3.2 \mathrm{~kg}$ were used for this study. The rabbits were named from $A$ to $D$ and fed a normal laboratory diet (LabDiet, PMI Feed Co., USA) before experiment. Animal experiments were carried out humanely, in conformity with the "Guide for the Care and Use of Laboratory Animals" enunciated by School of Medicine, Hokkaido University in 1988.

\section{2 Procedures for creating a moderate stenosis in arteries}

Rabbits were sedated with ketamine hydrochloride by injecting the drug intramuscularly and then anesthetized with sodium pentobarbital by injecting intravenously $(0.4 \mathrm{ml})$, and maintained under anesthesia during the whole procedures by further admin- istration of the anesthetic $(0.2-0.4 \mathrm{ml}$ every $30 \mathrm{~min}$ utes). Then under sterile conditions, median incision was made on the neck and approximately $5 \mathrm{~cm}$ long segments of bilateral common carotid arteries were exposed as carefully as possible to minimize damages to muscular tissues and microvessels. To release the tension of the artery caused by exposing the artery, a solution of papaverine hydrochloride was sprinkled on the blood vessel, and then a moderate stenosis was created at one or two locations in both the right and left common carotid arteries by winding a 6-0 suture thread twice on them. Then incised muscle layers and skin tissues were sewed up to their original positions with a 3-0 silk suture to complete the operation.

\section{3 Harvesting arteries}

The rabbits which underwent surgical procedures were kept alive for 8 weeks $(n=2)$ and 14 weeks $(n=2)$ postoperatively for recovery and healing, and then they were brought back to the operating room for harvesting the arteries. During the above period, they were fed a diet containing $0.2 \%$ cholesterol at a rate of $100 \mathrm{~g}$ per day, and the cholesterol level in blood was monitored every week by sending blood samples taken from the rabbits to a private test center (Shiroishi Rinsho Shiken Co., Sapporo).

The arteries were harvested as follows. Under general anesthesia with sodium pentobarbital, incision was made on the neck, and an approximately $5 \mathrm{~cm}$ long segment of both the left and right common carotid arteries containing the segment where stenoses have been created was reexposed. Heparin ( $400 \mathrm{U} / \mathrm{kg}$ body weight) was administered intravenously to prevent blood coagulation, and the rabbits were killed by overdosing sodium pentobarbital. Then, an approximately $4 \mathrm{~cm}$ long segment containing the stenosis at a mid portion of it was cannulated at both ends with stainless steel pipes and flushed with isotonic saline solution to wash out the blood. The length of the arterial segment was measured accurate$l y$, and then the artery was isolated from the body of the animal, and was fixed on a solid stainless steel frame keeping its length measured in vivo. Finally, the isolated arterial segment was histologically fixed by immersing in and perfusing it with a $4 \%$ formalde hyde-phosphate buffer solution $(\mathrm{pH}=7.4)$ at a per fusion pressure of $13.3 \mathrm{kPa}(100 \mathrm{mmHg})$ over a night.

\section{4 Preparation of histological specimens}

The arterial segment fixed histologically was divided into short ringed segments by cutting the vessel every $5 \mathrm{~mm}$ starting from a location $2.5 \mathrm{~mm}$ distal to the apex of the stenosis where a suture thread was wound. Each of the ringed segments was numbered and embedded in paraffin so that the crosssection of the blood vessel faced to the cutting surface 
of a paraffin block. Then, several histological specimens with a thickness of $5 \mu \mathrm{m}$ were prepared from each block by slicing it continuously from the surface where the tissue sample of the vessel appeared first and counting the number of the slices. The locations of the prepared specimens in the longitudinal direction of the whole arterial segment were obtained from the section and slice numbers taking account of shrinkage of the blood vessel which occurred during the preparation of the histological specimens. After the histological specimens were fully extended and dried on a slide glass, it was stained with hematoxylin-eosin and van Gieson elastica stains for observations under a transmission light microscope.

\subsection{Analysis of histological specimens}

The histological specimens were observed under a light microscope (MICROPHOT-FX, Nikon Co., Tokyo) and then the images photographed with a digital camera (DP10, Olympus Optical Co., Ltd., Tokyo) were recorded in a personal computer. Locations where intimal thickening occurred in the stenosed artery were specified by checking each histological specimen. For the specimens in which intimal thickening was observed, the maximum thickness of the intima in each specimen was measured using an image processor (Image Tool Ver. 2.0, UTHSCSA, USA).

Inner diameter of the vessel was also obtained by measuring the length of the outline of the luminal surface of the vessel and dividing it by the circular constant. Measurements were carried out over the whole length of the stenosed artery, and a mean diameter at a location proximal to the stenosis, $d_{0}$, and a minimum diameter at the apex of the stenosis, $d_{s}$, were obtained. Then, a degree of stenosis, $\gamma$, defined as $\gamma=\left(d_{0}-d_{s}\right) / d_{0}$, was calculated.

\section{6 Preparation of Sudan IV stained aortas}

In order to find out the effect of feeding a cholesterol-rich diet to the rabbits on the formation of lipid rich plaques on their arterial wall, a portion of the aorta which encompassed from the ascending aorta to the common iliac artery was isolated from the body of the same animal used for the above experiments. After opening the vessel by cutting longitudinally and washing out the blood, the vessel was fixed with a $4 \%$ formaldehyde-phosphate buffer solution, and then stained with Sudan IV by immersing it in Sudan IV solution for 1 hour and in a 50\% ethanol solution for several minutes.

\section{Computational Study}

\subsection{A model of stenosed arteries used for computational analyses}

In order to investigate the effects of a moderate stenosis created in the common carotid artery of the rabbit on the localization of intimal thickening, we calculated the distributions of wall shear stress and LDL concentration at the luminal surface of the blood vessel by a computer simulation of blood flow and transport of LDL through a stenosed artery using an axisymmetric model of the vessel shown in Fig. 1. The model was constructed by creating a cosineshaped axisymmetric stenosis in a straight cylindrical artery based on the vessel diameter, $d_{0}$ and the degree of stenosis obtained from animal experiments. It was assumed that the length of the stenosed segment and the two straight segments proximal and distal to that were $d_{0}, 22 d_{0}$, and $5 d_{0}$, respectively.

\section{2 Computational analysis of blood flow}

Under the assumptions that arterial wall is rigid, blood is an incompressible Newtonian fluid with a density of $1.05 \mathrm{~g} / \mathrm{cm}^{3}$ and a viscosity of 0.035 $\mathrm{g} /(\mathrm{cm} \cdot \mathrm{s})$, and blood flow is steady, the Navier-Stokes equation which were expressed using axisymmetric two dimensional coordinate system $(z, r)$ with its origin at the center of the stenosis as shown in Fig. 1 was solved together with the equation of continuity to obtain wall shear stresses. Boundary conditions applied here were a parabolic velocity profile at the inlet, a constant pressure at the outlet, and axisymmetric conditions on all the variables at the central axis. Taking the water filtration flow at the vessel wall into consideration, an water filtration velocity directed normal to the wall and having a magnitude of $V_{w}$ was given as two components of the velocity vector at the vessel wall.

\section{3 Computational analysis of LDL transport}

The mass transport of LDL in blood flowing in steady flow through a straight artery containing an axisymmetric stenosis can be described by

$$
u \frac{\partial C}{\partial z}+v \frac{\partial C}{\partial r}-D\left(\frac{1}{r} \frac{\partial C}{\partial r}+\frac{\partial^{2} C}{\partial r^{2}}\right)=0
$$

where $C$ is the concentration of LDL, $D$ is the diffusivity of LDL, and $u$ and $v$ are velocity components in the axial and radial direction, respectively. The diffusivity of $\mathrm{LDL}$ in blood at $37^{\circ} \mathrm{C}$ was estimated from Stokes-Einstein equation to be $5 \times 10^{-6} \mathrm{~mm}^{2} / \mathrm{s}$. Boundary conditions applied were a uniform and a constant concentration of LDL at the inlet, zero gradi-

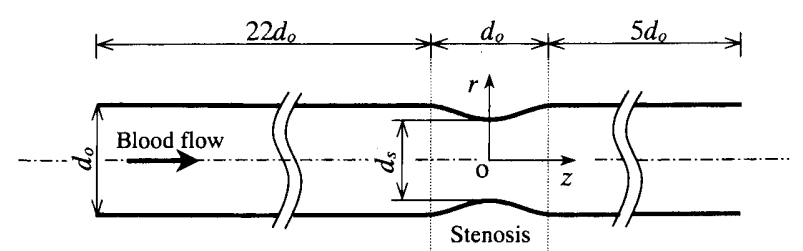

Fig. 1 A model of a moderately stenosed artery used for the computational study 
ent of LDL concentration in the longitudinal direction at the outlet, axisymmetric condition at the central axis, and conservation of mass among the LDL passing through a semipermeable wall, moving toward the vessel wall by a filtration flow, and diffusing back to the mainstream, at the vessel wall as described by

$$
C_{w} V_{w}-D \frac{\partial C}{\partial n}=\phi V_{w} C_{w}
$$

where $C_{w}$ is the surface concentration of LDL, $n$ is a coordinate normal to the wall, and $\phi$ is the penetration ratio of LDL at the vessel wall which was estimated to be 0.005 from the permeability of an arterial wall to $\mathrm{LDL}^{(13)}$.

\section{4 Procedures for computation}

Under the assumption that LDL molecules move together with fluid elements and the viscosity of blood remains constant even when the surface concentration of LDL changed several ten percent, the governing equations of flow were solved numerically using a simulation software (Ansys-Flotran Ver.5.6, distributed by Cybernet Systems Co. Ltd., Tokyo) to obtain the fluid velocity. Then, by substituting the value of fluid velocity into Eq. (1), the equation was solved using an in-house program code based on Petrov-Galerkin formulations for finite element method with a streamline upwind technique ${ }^{(16)}$ to obtain the concentration of LDL. To solve the Navier-Stokes equation, the solution domain of the stenosed artery was divided into 60 elements of gradually diminishing size in the radial direction and 800 elements in the longitudinal direction, assigning fine meshes to the stenosed segment. In order to obtain a stable solution with desired accuracy in the calculation of LDL concentration, the elements at locations greater than $90 \%$ of the inner radius of the vessel at any crosssection were further divided into 4 subelements in the radial direction. The fluid velocity at the newly created node was obtained by carrying out linear interpolation.

Wall shear stress was evaluated at each node on the vessel wall by calculating the velocity gradient in the direction normal to the vessel wall from velocity vectors parallel to the wall at the nodes which constituted the element closest to the vessel wall and multiplying it by the viscosity of blood.

\section{Results}

\section{1 Experimental results}

4.1.1 Cholesterol level in blood and staining of the arterial wall with Sudan IV The cholesterol level in blood increased gradually in two weeks postoperatively and then attained stable values except the case of rabbit D. Mean cholesterol level of rabbit A, $\mathrm{B}$, and $\mathrm{C}$ evaluated at the time of harvesting their common carotid arteries at 8 weeks (A, B) and 14 weeks (C) postoperatively was $195 \pm 49 \mathrm{mg} / \mathrm{dl}$ (Mean \pm S.D.) which was about 10 times higher than that $(19 \pm 4 \mathrm{mg} / \mathrm{dl})$ obtained before starting cholesterol feeding. The cholesterol level of rabbit D continued to increase and finally reached an extremely high value of $910 \mathrm{mg} / \mathrm{dl}$.

From the observation of the luminal surface of the aorta stained with Sudan IV, it was found that lipid-rich plaques formed only in the aorta of rabbit D. Therefore, it was confirmed that the cholesterol levels of rabbit $\mathrm{A}, \mathrm{B}$, and $\mathrm{C}$ did not reach an abnormally high level which causes the formation of a lipid-rich plaque and intimal thickening everywhere in the artery.

4.1.2 Histological changes Nine arterial specimens containing a moderate stenosis were successfully obtained from the common carotid arteries of four rabbits. Figure 2 shows photographs of hematoxylin-eosin-stained histological specimens of the vessel wall taken from two different locations that were right proximal and distal to the apex of a stenosis created on the left common carotid artery of rabbit $\mathrm{C}$. As shown in Fig. 2(a), the intima of the vessel wall located proximal to the apex of the stenosis where flow was not expected to be disturbed by the presence of a stenosis was composed of a monolayer of endothelial cells directly adherent to the internal elastic

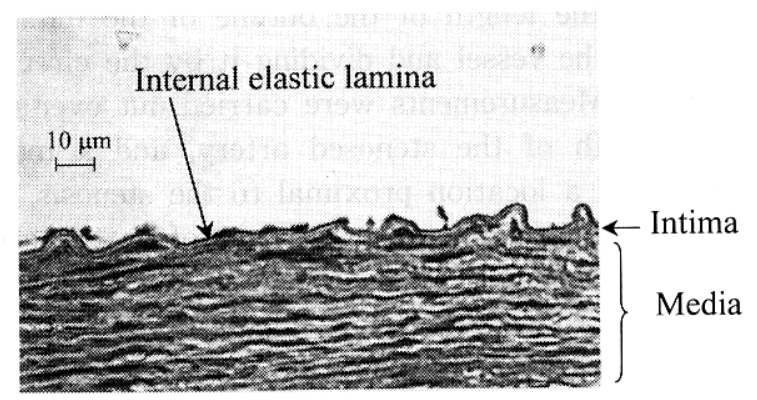

(a) An arterial wall with no intimal thickening (proximal side away from the stenosis)

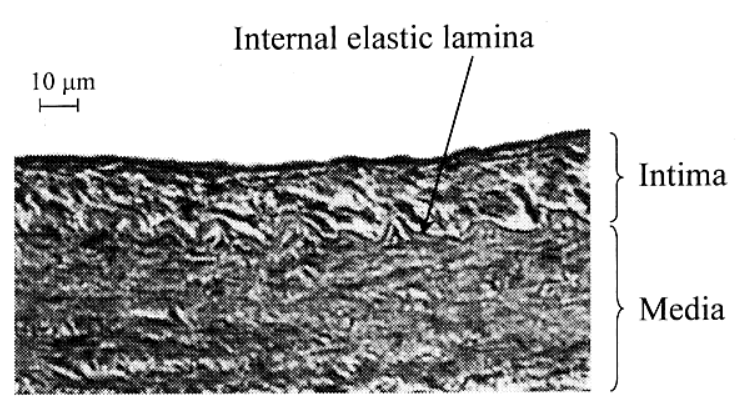

(b) An arterial wall with intimal thickening (distal side near the apex of the stenosis)

Fig. 2 Photographs of hematoxylin-eosin stained histological specimens prepared from the rabbit common carotid artery containing a moderate stenosis 
Table 1 Geometric data on the stenosis created in the common carotid artery of the rabbit

\begin{tabular}{|c|c|c|c|c|c|c|c|c|c|}
\hline \multirow[b]{2}{*}{ Specimen } & \multirow[b]{2}{*}{ Rabbit } & \multirow[b]{2}{*}{ Artery } & \multirow[b]{2}{*}{$\begin{array}{c}\text { Period } \\
\text { [weeks] }\end{array}$} & \multirow{2}{*}{$\begin{array}{l}\text { Chol. conc. } \\
{[\mathrm{mg} / \mathrm{dl}]}\end{array}$} & \multirow{2}{*}{$\begin{array}{l}\text { Diameter, } d_{\mathrm{o}} \\
{[\mathrm{mm}]}\end{array}$} & \multirow[b]{2}{*}{$\begin{array}{c}\text { Stenosis, } \gamma \\
{[\%]}\end{array}$} & \multicolumn{3}{|c|}{ Intimal thickening } \\
\hline & & & & & & & Side & $\begin{array}{l}\text { Length } \\
{[\mathrm{mm}]}\end{array}$ & $\begin{array}{c}\text { Max. thickness } \\
{[\mu \mathrm{m}]}\end{array}$ \\
\hline No. 1 & $\mathrm{~A}$ & left c.c. ${ }^{f t}$ & 8 & 188 & 1.60 & 20.7 & distal & 1.62 & 36.9 \\
\hline No. 2 & \multirow{3}{*}{ B } & right c.c.a & \multirow{3}{*}{8} & \multirow{3}{*}{248} & 1.64 & 24.6 & distal & 1.25 & 44.1 \\
\hline No. 3 & & right c.c.a & & & 1.64 & 15.0 & distal & 4.50 & 37.5 \\
\hline No. 4 & & left c.c.a & & & 1.96 & 24.1 & distal & 1.68 & 49.3 \\
\hline No. 5 & \multirow{2}{*}{$\mathrm{C}$} & right c.c.a & \multirow{2}{*}{14} & \multirow{2}{*}{151} & 1.86 & 39.3 & distal & 1.66 & 18.4 \\
\hline No. 6 & & left c.c.a & & & 2.12 & 29.1 & distal & 2.29 & 22.7 \\
\hline No. 7 & \multirow{3}{*}{ D } & right c.c.a & & \multirow{3}{*}{910} & - & - & proximal & - & - \\
\hline No. 8 & & right c.c.a & 14 & & - & - & distal & - & - \\
\hline No. 9 & & left c.c.a & & & - & - & distal & - & - \\
\hline \multicolumn{2}{|c|}{ Mean S.D. ${ }^{\dagger}$} & & & $196 \pm 48$ & $1.80 \pm 0.21$ & $25.5 \pm 8.5$ & & $1.61 \pm 0.78$ & $34.8 \pm 12.0$ \\
\hline
\end{tabular}

lamina, and no intimal thickening was observed. In contrast to this, as shown in Fig. 2(b), the intima of the vessel wall located distal to the apex of the stenosis where flow was expected to be disturbed by the presence of the stenosis was composed of a monolayer of endothelial cells, the internal elastic lamina, and some cellular substances between them, which was noticed by the presence of several stained nuclei, making the intima thicker than that at a proximal site. These results indicated that creation of a moderate stenosis in the rabbit common carotid artery caused intimal thickening which was easily detectable under a light microscope.

Intimal thickening was found at the distal side of the stenosis in eight of nine arterial specimens. The site was restricted in a region which extended from the apex of the stenosis to the distal end of the stenosis. Table 1 shows the period in which the animals were kept alive postoperatively, the side of the common carotid artery used to prepare histological specimens, the concentration of cholesterol in blood measured at the time of harvesting the arteries, the degree of stenosis, the location and the length of the segment where intimal thickening occurred, and the maximum thickness of the intima obtained in the segment. The specimens from No. 7 to No. 9 which were obtained from rabbit $D$ with a high concentration of cholesterol in blood at the time of harvesting the artery were examined whether the intimal thickening developed proximally or distally to the stenosis because formation of lipid plaques with intimal thickening occurred everywhere in the artery due to hypercholesterolemia. Although there were only a few data available, there was a tendency that the maximum thickness of the intima increased with increasing the concentration of cholesterol.

\subsubsection{Distribution of intimal thickness Figure} 3 shows the distribution of mean intimal thickness in the region of intimal thickening obtained from all the arterial specimens. The abscissa of this figure indi-

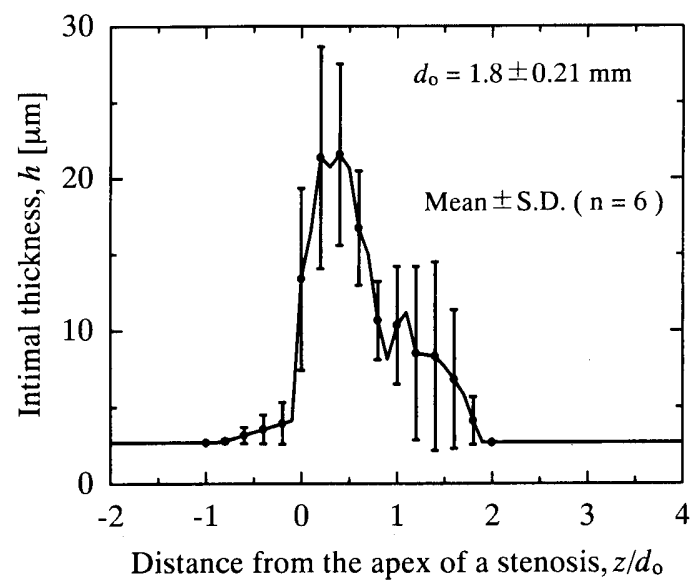

Fig. 3 Plots of the intimal thickness against the distance from the apex of the stenosis

cates the distances from the apex of the stenosis in the proximal and distal directions that were normalized by a vessel diameter, $d_{0}$, and the negative sign indicates proximal to the stenosis. Here, the mean values of intimal thickness were obtained as follows. Firstly, in all the arterial specimens, the distance from the apex of a stenosis was normalized by the diameter of each arterial specimen. Next, the thickness of the intima was calculated at an interval of $0.1 d_{0}$ starting from the apex of the stenosis by linearly interpolating the thickness from the values measured at several arbitrary locations in each specimen. Then at each location, a mean value of the intimal thickness of all the arterial specimens and its standard deviation were calculated. In this calculation, the thickness of the intima at all the locations where no intimal thickening occurred was assumed to be constant at $2.7 \mu \mathrm{m}$. As evident from this figure, the thickness of the intima sharply increased at a location right distal to the apex of the stenosis, taking the largest value at $z / d_{0}=0.4$, and then decreased to attain a constant value which was $2.7 \mu \mathrm{m}$ at $z / d_{0}=2$. 


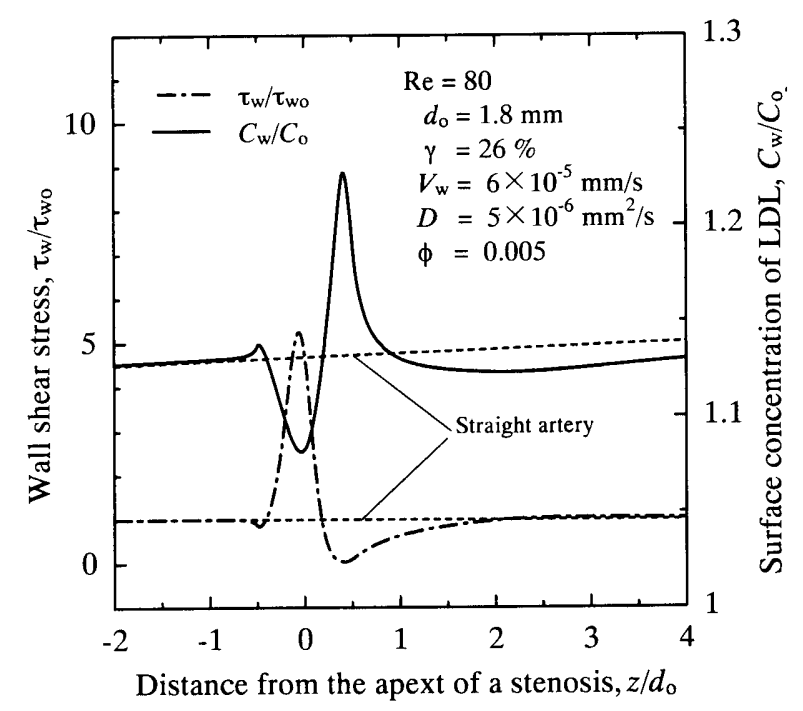

Fig. 4 Distributions of wall shear stress and surface concentration of LDL in an artery containing a moderate stenosis

\section{2 Computational results}

4.2.1 Distributions of $\boldsymbol{\tau}_{w}$ and $\boldsymbol{C}_{w}$ in an artery containing a moderate stenosis Computer simulations of blood flow and LDL transport through stenosed arteries were carried out using the vessel diameter at the inlet $\left(d_{0}=1.8 \mathrm{~mm}\right)$ and the degree of stenosis $(\gamma=26 \%)$ which were obtained in the present experiments (Table 1). It was assumed that the Reynolds number at the inlet, $R e$, was 80 which was estimated from the mean blood flow rate in the common carotid artery of the rabbit reported in the literature $^{(17)}$ and the water filtration velocity at the arterial wall, $V_{w}$, was $6.0 \times 10^{-5} \mathrm{~mm} / \mathrm{s}$ which was obtained in our previous experimental study ${ }^{(18)}$. Under these flow conditions, since the degree of stenosis was not severe enough to cause flow separation, fluid elements just traveled through the stenosed artery in one direction, and no recirculation flow occurred in the vessel. The wall shear stress evaluated at the inlet, $\tau_{w 0}$, was $2.3 \mathrm{~Pa}$.

Figure 4 shows the distributions of wall shear stress, $\tau_{w}$, and LDL concentration at the luminal surface of the blood vessel, $C_{w}$, which were normalized by the values at the inlet, $\tau_{w 0}$ and $C_{0}$, respectively. The abscissa indicates the distance from the apex of the stenosis which was normalized by the vessel diameter at the inlet, $d_{0}$. The dashed lines in this figure show the values calculated for a straight artery having the same diameter as that of the inlet portion of the stenosed artery.

As shown in Fig. 4, wall shear stress slightly decreased at the leading edge of the stenosis and then sharply increased to a value 5.2 times higher than the baseline value for a straight artery or non-constricted portions upstream of the stenosis. On the distal side

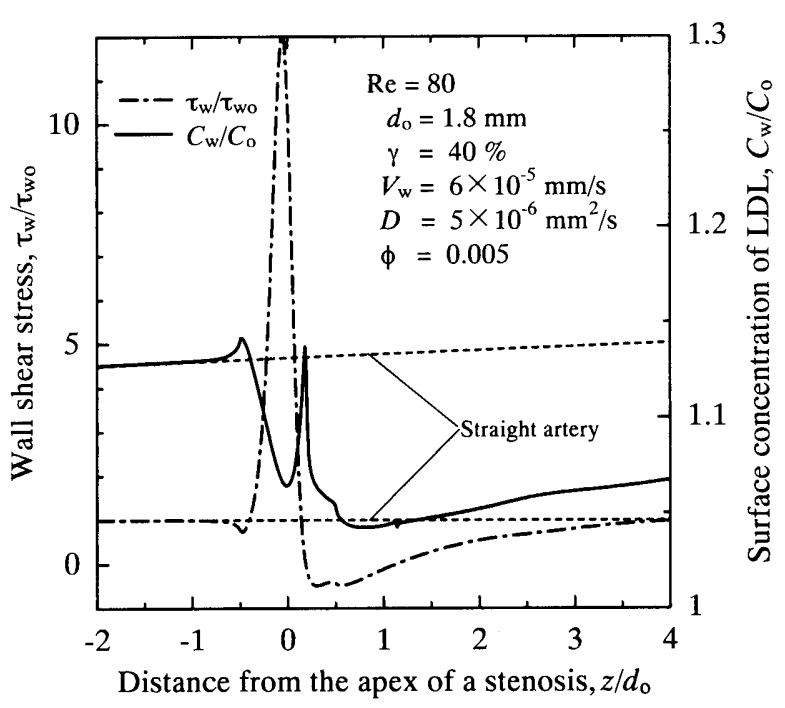

Fig. 5 Distributions of wall shear stress and surface concentration of LDL in an artery containing a severe stenosis

of the stenosis, it decreased very sharply at the receding portion of the stenosis and took a minimum which was lower than the baseline value and nearly zero at a location close to the receding edge of the stenosis. It then increased gradually and attained to the baseline value at $z / d_{0}=2$.

The surface concentration of LDL kept increasing from the inlet of the artery due to the occurrence of concentration polarization of $\mathrm{LDL}$, reaching to a value which was $11.3 \%$ higher than that at the inlet at $z / d_{0}=-2$. Then in the region of the stenosis, it increased or decreased in phase with the change in wall shear stress, but in opposite directions. Thus it showed the lowest value at a location slightly upstream of the apex of the stenosis where wall shear stress took the highest value, and the highest value at a location close to the receding edge of the stenosis where wall shear stress took the lowest value. The highest concentration which occurred at $z / d_{0}=0.4$ was $23 \%$ higher than that at the inlet. The surface concentration of LDL then decreased and attained to a value slightly lower than that for a straight artery at $z / d_{0}=$ 2 where wall shear stress restored a baseline value.

\subsubsection{Distributions of $\tau_{w}$ and $C_{w}$ in an artery} containing a severe stenosis Figure 5 shows the distributions of wall shear stress and surface concentration of LDL which were obtained from the calculation for blood flowing at $R e=80$ through a severely stenosed artery with a degree of stenosis, $\gamma=40 \%$. In this case, the blood flow in the stenosed artery was greatly disturbed by the occurrence of flow separation at $z / d_{0}=0.19$ which was just distal to the apex of the stenosis, and formation of an annular vortex between the point of flow separation and the point of reattach- 
ment located at $z / d_{0}=1.13$ where wall shear stress took negative values. Comparison of these results with those shown in Fig. 4, it was found that the effect of flow disturbance is much greater on the distribution of surface concentration of LDL than on the distribution of wall shear stress because the concentration boundary layer was destroyed by flow separation and formation of an annular vortex. The region where wall shear stress was lower than the baseline value was extended further distally by increasing the degree of stenosis, but the shape was similar to the case of a moderate stenosis. In contrast to this, although the surface concentration was locally elevated just distal to the stenosis, taking a local maximum at the point of flow separation where wall shear stress was zero, the value was not so high and comparable to the baseline value for a straight artery. Furthermore, the surface concentration sharply decreased after taking a maximum and settled down to a value far below the surface concentration of LDL in a straight artery at $z / d_{0}=4$ where wall shear stress recovered the base line value.

4.2.3 Effects of $R e$ and the degree of stenosis As shown above, the surface concentration of LDL in the stenosed artery was strongly affected by flow disturbance which was augmented by increasing $R e$ or the degree of stenosis. Hence, we carried out calculations of LDL surface concentration by varying $R e$ and the degree of stenosis, and found out the highest value which appeared in the receding portion of the stenosis and the lowest value following it from the distribution of surface concentration of LDL as two characteristic values.

Figure 6 shows the plots of the highest and the lowest values in the surface concentration of LDL against $R e$ obtained by keeping the degree of stenosis at $26 \%$. The results obtained for arteries having various degree of stenosis by keeping $R e$ at 80 is shown in Fig. 7. The concentrations shown in these figures were normalized by the value of LDL surface concentration in a straight artery, $\bar{C}_{w}$, evaluated at two locations which corresponded to the sites where the surface concentration of LDL took the highest and the lowest values in the stenosed artery. The dashed lines indicate the threshold of $\operatorname{Re}$ and $\gamma$ for the occurrence of flow separation in the stenosed artery. It was found that the highest value in surface concentration of LDL increased with increasing $R e$ and the degree of stenosis until flow separation occurred, indicating that concentration polarization of LDL in the stenosed artery was maximized under a condition critical for the occurrence of flow separation. Comparison of the results given in Fig. 6 with those in Fig. 7 shows that the surface concentration of LDL is affected more

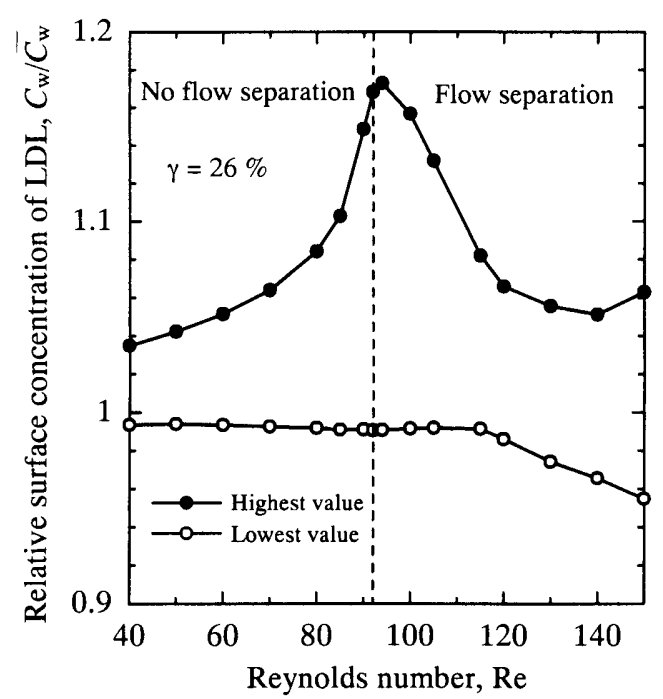

Fig. 6 Plots of the highest and the lowest values in the surface concentration of LDL in the region distal to the apex of the stenosis against the Reynolds number

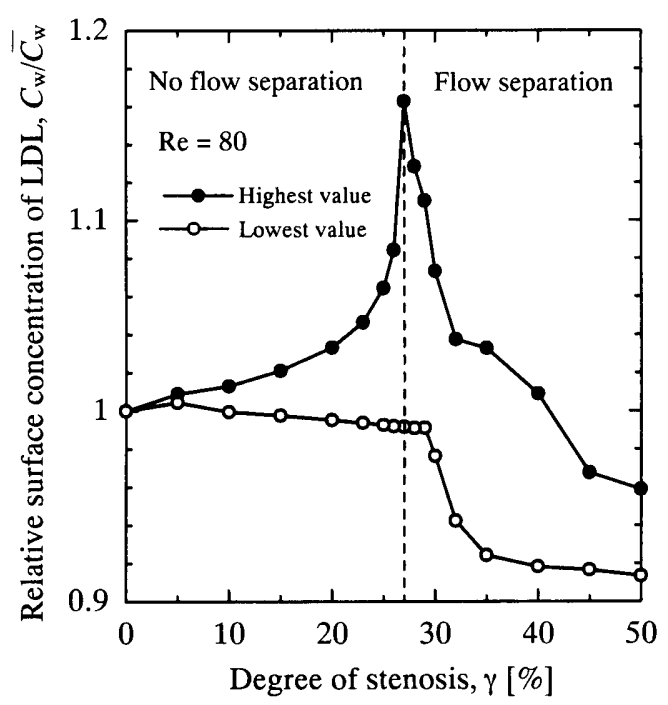

Fig. 7 Plots of the highest and the lowest values in the surface concentration of LDL in the region distal to the apex of the stenosis against the degree of stenosis

strongly by the degree of stenosis than $R e$.

4. 2.4 Degree of flow disturbance in the stenosed artery In Fig. 8, the relationship between critical $R e$ for the occurrence of flow separation in stenosed arteries and the degree of stenosis is shown by a solid line. The square symbols in the figure show experimental data calculated based on the flow rate $(22$ $\left.\mathrm{cm}^{3} / \mathrm{min}\right)^{(17)}$ and the diameter of each artery. As evident from the figure, most of the experimental data were located in the area of no flow separation except one specimen (No. 5) and close to the solid line which shows the critical conditions for the occurrence of 


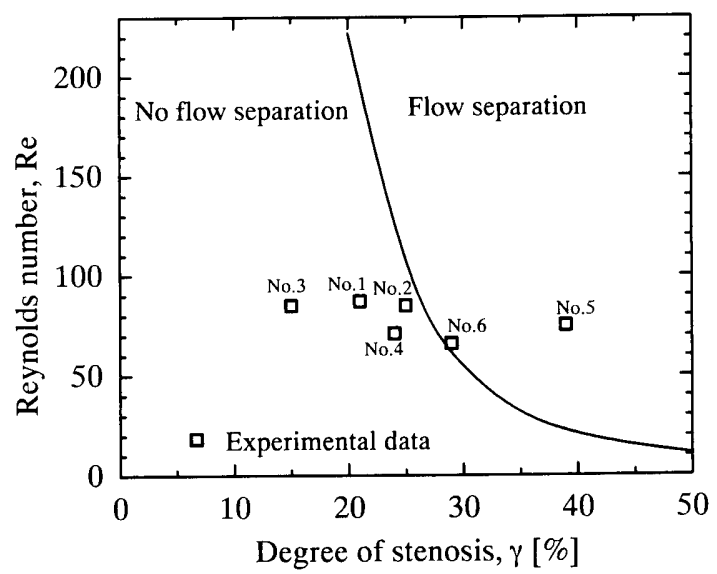

Fig. 8 Plots of the critical Reynolds number for the occurrence of flow separation in the stenosed artery against the degree of stenosis. The data indicated by square symbols were obtained from the present experimental study.

flow separation.

\section{Discussion}

Through the animal experiments carried out in the present study, it was found that creation of a moderate stenosis which caused a 15 to $39 \%$ reduction in diameter in the common carotid arteries of the rabbit induced intimal thickening in a very narrow restricted region located right distal to the apex of the stenosis. If the intimal thickening occurred as some biological responses to an injury caused by the suture thread wound on the artery, and/or elevation of cholesterol level in blood caused by feeding a cholesterolrich diet, it would have occurred equally on both proximal and distal sides of the stenosis. Furthermore, since it has been shown that in the case of the rabbits fed a cholesterol-rich diet, atheromatous plaques develop everywhere in the aorta if the concentration of cholesterol in blood is elevated to a level which is extremely high ${ }^{(19)}$, we kept monitoring the concentration of cholesterol in blood during the healing period of the rabbits which underwent a surgical procedure so that it did not reach such a high level. Therefore, we considered that the factor which played a key role in the localized genesis and development of intimal thickening in the present study is the disturbed flow as in the case of atherosclerosis which develops preferentially at certain sites in the human arterial system where flow is disturbed and wall shear stress is relatively $\operatorname{low}^{(6)-(10)}$.

Our computational study on blood flow and transport of LDL through a stenosed artery carried out by computer simulations revealed that the disturbed flow generated at the stenosis greatly affected the distribution of wall shear stress and LDL concentration at the luminal surface of the stenosed artery in a region which extended from the leading edge of the stenosis to a site further downstream of the receding edge of the stenosis (Fig. 4). By comparing the results with that of the intimal thickness shown in Fig. 3, it was found that intimal thickening occurred at a site distal to the stenosis where wall shear stress was relatively low. In this sense, localization of intimal thickening well resembles that of atherosclerosis as mentioned above. An important finding was made through our computational analysis that the surface concentration of LDL was locally elevated at the receding portion of the stenosis where intimal thickening developed, and that the location where the arterial intima was thickened most well corresponded to the site where wall shear stress was the lowest and surface concentration of LDL was the highest. Since it has been shown that elevation of LDL level in blood increases its uptake by arterial wall, leading to development of intimal thickening and atherosclerotic changes in the wall ${ }^{(20)-(23)}$, these results strongly suggest that blood flow plays an important role in the localized genesis and development of intimal thickening in stenosed arteries by locally augmenting concentration polarization of LDL which occurs at a blood/endothelium boundary.

Our computational studies also showed that the distribution of surface concentration of LDL in the region distal to the stenosis becomes quite different depending on whether flow separation occurs or not in the stenosed artery because if flow separation occurs, the flow downstream of the stenosis is disturbed and the concentration boundary layer is destroyed. Thus, we examined whether flow separation occurred or not in those stenosed arteries used in the present study by plotting the degree of stenosis and a corresponding Reynolds number on a map which indicated the area where flow separation occurred and the area where no flow separation occurred by dividing by a line which showed the critical Re for the occurrence of flow separation as a function of the degree of stenosis (c.f. Fig. 8). It was found that in most of the stenosed arteries, flow separation did not occur, but the condition was very close to a critical state to cause flow separation. As shown in Figs. 6 and 7, under such a flow condition, the surface concentration of LDL in the stenosed artery is elevated to the highest level. Therefore, it is likely that this helped the uptake of LDL by endothelial cells, leading to localized development of intimal thickening in a region of high LDL surface concentration right distal to the apex of the stenosis.

In our calculations, it was assumed that the blood flow in the stenosed artery was steady. Thus, the results on the distribution of LDL surface concentra- 
tion obtained in the present study may not apply as they are to an artery in vivo where flow is pulsatile. However, as shown in Fig. 6, since the range of the Re which causes moderate concentration polarization of LDL in a stenosed artery is relatively wide and extends beyond the $R e$ which causes flow separation, the concentration boundary layer formed distal to the apex of the stenosis will not be destroyed easily even if the $R e$ varies within the range of $R e$ shown in this figure. In fact, it is predicted from the velocity profile measured in the common carotid artery of a dog that, in pulsatile flow, the Reynolds number varies only from $60 \%$ to $190 \%$ of the mean value ${ }^{(24)}$. Therefore, it is very likely that concentration polarization of LDL still occurs in moderately stenosed arteries in vivo.

It has been reported that in experimental animals, creation of a moderate and severe axisymmetric stenosis in an artery causes changes of opposite nature in the structure of the vessel wall in a restricted region around the stenosis, i.e., thickening by the development of intimal hyperplasia and thinning by the development of poststenotic dilatation, respectively ${ }^{(12),(25),(26)}$. In our experiments, enlargement of vessel diameter distal to the stenosis was observed only in the arterial specimen No.5 (data are not shown in this paper) in which flow separation was expected to occur from the results shown in Fig. 8. If we consider the above phenomena in the light of the results of our present analyses, it is very likely that thickening and thinning of the vessel wall which occur following partial occlusion of an artery by the creation of a moderate and severe axisymmetric stenosis, respectively, are caused by the change in surface concentration of LDL (an important nutriment for division and proliferation of all the cells forming the vessel wall) since its excessive and short supply may result in the hypertrophy and hypotrophy of the cells of the vessel wall, respectively.

\section{Conclusions}

In the present study, we investigated experimentally the precise locations and the distributions of intimal thickening which was caused by the creation of a moderate stenosis in the common carotid artery of the rabbit and also theoretically the characteristics of the flow and transport of LDL in stenosed arteries based on the data obtained experimentally in order to find an answer to a question why a locally disturbed flow induces the localization of intimal thickening. As the results, it was confirmed that intimal thickening occurred and developed preferentially in a narrow and restricted region distal to a stenosis where wall shear stress was relatively low as it did so in the cases of atherosclerosis and intimal hyperplasia. Next, it was found that concentration polarization of LDL occurred at the blood/endothelium boundary in the stenosed artery, and this created a region of high LDL concentration at the luminal surface of the artery in the receding portion of the stenosis where wall shear stress was low and intimal thickenings developed preferentially. Furthermore, it was found that the location where surface concentration of LDL took the highest value well corresponded to the location where the intima was the thickest and wall shear stress was the lowest. These results strongly suggest that blood flow plays an important role in the localized path. ogenesis and development of intimal thickening by locally augmenting concentration polarization of LDL which occurs at a blood/endothelium boundary.

\section{Acknowledgements}

We thank Mr. Takahide Ishizaka of the Research Institute for Electronic Science, Hokkaido University and Mr. Toshifumi Harada of Graduate School of Hokkaido University for their help in experimental studies. This work was supported by a Grant-in-Aid for Scientific Research (No. 11680831) from the Ministry of Education, Science and Culture of Japan.

\section{References}

(1) Stary, H.C., Chandler, A.B., Glagov, S., Guyton, J.R., Insull, W.Jr., Rosenfeld, M.E., Schaffer, A., Schwartz, C.J., Wagner, W.D. and Wissler, R.W., A Definition of Initial, Fatty Streak, and Intermediate Lesions of Atherosclerosis: A Report from the Committee on Vascular Lesions of the Council on Arteriosclerosis, American Heart Association, Arterioscler Thromb., Vol. 14 (1994), pp. 840-856.

(2) Getz, S., The Involvement of Lipoproteins in Atherogenesis Evolving Concepts, Ann. N.Y. Acad. Sci., Vol. 598 (1990), pp. 598: 17-28.

( 3 ) Fox, B., James, K., Morgan, B. and Seed, A., Distribution of Fatty and Fibrous Plaques in Young Human Coronary Arteries, Atherosclerosis, Vol. 41 (1982), pp. 337-347.

(4) Grøttum, P., Svindland, A. and Walløe, L., Localization of Atherosclerotic Lesions in the Bifurcation of the Main Left Coronary Artery, Atherosclerosis, Vol. 47 (1983), pp. 55-62.

(5) Svindland, A., Localization of Atherosclerotic Lesions in Three Cerebral Arterial Bifurcations, Acta. Path. Microbiol. Immunol. Scand., Sect. A92 (1984), pp. 177-183.

(6) Caro, C.G., Fitz-Gerald, J.M. and Schroter, R.C., Arterial Wall Shear and Distribution of Early Atheroma in Man, Nature, Vol. 223 (1969), pp. 1159-1161.

(7) Zarins, C.K., Giddens, D.P., Bharadvaj, B.K., Sottiurai, V.S., Mabon, R.F. and Glagov, S., Carotid Bifurcation Atherosclerosis: Quantitative Correlation of Plaque Localization with Flow 
Velocity Profiles and Wall Shear Stress, Circ. Res., Vol. 53 (1983), pp. 502-514.

(8) Ku, D.N., Giddens, D.P., Zarins, C.K. and Glagov, S., Pulsatile Flow and Atherosclerosis in the Human Carotid Bifurcation : Positive Correlation between Plaque Location and Low and Oscillating Shear Stress, Atherosclerosis, Vol. 5 (1985), pp. 293-302.

(9) Motomiya, M. and Karino, T., Flow Patterns in the Human Carotid Artery Bifurcation, Stroke, Vol. 15 (1984), pp. 50-56.

(10) Asakura, T. and Karino, T., Flow Patterns and Spatial Distribution of Atherosclerotic Lesions in Human Coronary Arteries, Circ. Res., Vol. 66 (1990), pp. 1045-1066.

(11) Ishibashi, H., Sunamura, M. and Karino, T., Flow Patterns and Preferred Sites of Intimal Thickening in End-to-end Anastomosed Vessels, Surgery, Vol. 117 (1995), pp. 409-420.

(12) Bomberger, R.A., Zarins, C.K. and Glagov, S., Subcritical Stenosis Enhances Distal Atherosclerosis, J. Surg. Res., Vol. 30 (1981), pp. 205-212.

(13) Wada, S. and Karino, T., Theoretical Study on Flow-dependent Concentration Polarization of Low Density Lipoproteins at the Luminal Surface of a Straight Artery, Biorheology, Vol. 36 (1999), pp. 207-223.

(14) Wada, S. and Karino, T., Relationship between Wall Shear Stress and Surface Concentration of Lipoproteins Calculated for a Multiple Bend of the Human Right Coronary Artery, ASME Proc. of Bioeng. Conf., BED-Vol. 42 (1999), pp. 735-736.

(15) Naiki, T. and Karino, T., Flow-dependent Concentration Polarization of Plasma Proteins at the Luminal Surface of a Semipermeable membrane, Biorheology, Vol. 36 (1999), pp. 243-256.

(16) Brooks, A.N., Streamline Upwind/Petrov-Galerkin Formulations for Convection Dominated Flows with Particular Emphasis on the Incompressible Navier-Stokes Equations, Comp. Meth. in Appl. Mech. and Eng., Vol. 32 (1982), pp. 199259.

(17) Song, R.H., Kocharyan, H.K., Fortunato, J.E., Glagov, S. and Bassiouny, H.S., Increased Flow and Shear Stress Enhance in vivo Transforming Growth Factor-beta 1 after Experimental Arterial Injury, Arterioscler. Thromb. Vasc. Biol., Vol. 20 (2000), pp. 923-930.
(18) Wada, S., Kaichi, M. and Karino, T., Effect of Water Filtration Velocity on Changes in Vessel Structure of Rabbit Common Carotid Artery upon Removal of the Adventitia, (in Japanese), Proc. 76th JSME Fall Annual Meeting, No. 98-3 (1998), pp. 259-260.

(19) Roach, M.R., Fletcher, J. and Cornhill, J.F., The Effect of the Duration of Cholesterol Feeding on the Development of Sudanophilic Lesions in the Rabbit Aorta, Atherosclerosis, Vol. 25 (1976), pp. 1-11.

(20) Ross, R. and Harker, L., Hyperlipidemia and Atherosclerosis, Chronic Hyperlipidemia Initiates and Maintains Lesions by Endothelial Cell Desquamation and Lipid Accumulation, Science, Vol. 193 (1976), pp. 1094-1100.

(21) Hoff, H.F. and Wagner, W.D., Plasma Low Density Lipoprotein Accumulation in Aortas of Hypercholesterolemic Swine Correlates with Modifications in Aortic Glycosaminoglycan Composition, Atherosclerosis, Vol.61 (1986), pp. 231236.

(22) Schwenke, D.C. and Carew, T.E., Initiation of Atherosclerotic Lesions in Cholesterol-fed Rabbits, I. Focal Increases in Arterial LDL Concentration Precede Development of Fatty Streak Lesions, Arteriosclerosis, Vol. 9 (1989), pp. 895907.

(23) Baumann, D.S., Doblas, M., Daugherty, A., Sicard, G. and Schonfeld, G., The Role of Cholesterol Accumulation in Prosthetic Vascular Graft Anastomotic Intimal Hyperplasia, J. Vasc. Surg., Vol. 19 (1994), pp. 435-445.

(24) Okino, H., Kakiuchi, Y., Nishimura, A. and Sano, F., Pulsatile Blood Flow in the Arterial System, Resp. and Circ., (in Japanese), Vol. 14, No. 3 (1966), pp. 37-45.

(25) Roach, M.R., Poststenotic Dilatation in Arteries, In : Cardiovascular Fluid Dynamics, (ed. Bergel, D.H.), Vol. 2 (1972), pp. 111-139, Academic Press, New York.

(26) Kanegawa, Y., Murakami, T., Karino, T., The Changes in Vessel Diameter and Wall Structure that Occur Downstream of a Stenosis Created on the Common Carotid Artery of the Rabbit Fed on Cholesterol Rich Diet, (in Japanese), Technical Report of IEICE, MBE 98-34, (1998), pp. 59-66. 